\title{
A woman with a bamboo spine in the thoracic vertebra and normal sacroiliac joint; a 5-years undiagnosed ankylosing spondylitis: Case report and literature review
}

\author{
Masoud Mortezazadeh ${ }^{1}$, Farzan Vahedifard ${ }^{2}$, Sajjad Ahmadi-Renani ${ }^{3}$, Ahmad Salimzadeh ${ }^{1}$ \\ ${ }^{1}$ Sina Hospital, Tehran University of Medical Sciences, Tehran, Iran. ${ }^{2}$ Iran University of Medical Sciences, Tehran, Iran. \\ ${ }^{3}$ Tehran Heart Center, Tehran University of Medical Sciences, Tehran, Iran.
}

\begin{abstract}
Ankylosing spondylitis (AS) is a chronic inflammatory disease that causes deterioration in the function of the spine and peripheral joints. In addition to history and examination, imaging is important in diagnosing this disease. Pelvic X-rays in particular may show pseudowidening, erosion, and sclerosis sacroiliac joint. Spinal X-rays may also identify syndesmophytes. In more advanced stages, the spine may also be involved, forming a specific type of disease called "bamboo spine". The New York criteria, which includes radiologic and clinical criteria, are used to diagnose AS. The distribution of involvement in joints and bones in AS varies, but classically, it is ascending from the sacroiliac joint, lumbar, and thoracic region.

Herein, we report a case of AS that was undiagnosed for 5 years. Despite the normal appearance of the sacroiliac joint, severe involvement of the spinal column in the thoracic region known as "bamboo spine" was observed. The time order of bone involvement in this patient is contrary to what is usually seen.

Based on the New York criteria for AS, the case under discussion is not included in the AS definition; however, the patient had clinical symptoms of AS, bamboo spine, and showed a dramatic response to treatment of AS.

Heeding the course of the AS as well as the clinical signs and imaging results of various areas (heart, lumbar spine, and sacroiliac joint) will help physicians diagnose AS accurately and in a timely manner. Also, the radiologic manifestation of a rheumatologic disease may occur without any predictable order.
\end{abstract}

Keywords: Ankylosing spondylitis, bamboo spine, bone involvement, pathophysiology

\section{Introduction}

The term "spondyloarthropathy" refers to several often overlapping diseases that commonly produce inflammation in different areas of the body such as sacroiliac joints (sacroiliitis), axial spine (spondylitis), tendon, fascia, ligament insertion sites (enthesitis), oligoarthritis, rash (erythema nodusum), and uveitis. Because the rheumatoid factor is negative, the term seronegative spondyloarthritis has been used to refer to such cases in the past, which have included ankylosing spondylitis, psoriatic arthritis, inflammatory bowel disease arthritis, and reactive arthritis [1].

Ankylosing spondylitis (AS) is an inflammatory disease affecting various parts of the body, including the spine, peripheral joints, eyes, tendon, and cardiovascular system $[2,3]$. The initial symptoms of the AS include pain in the axial joints and limitation of movement. The pathogenesis of this disease is unclear, however, human leukocyte antigen B27 (HLA-B27) has been found in 90\% of AS patients [4]. The disease usually begins late in the second or third decade of life [5] and is reported to affect twice as many men as women [6].

Radiographic findings of the disease occur in a specific order: widening of the sacroiliac joint generally happens first, followed by erosion, sclerosis, and eventually ankylosis.

Inflammation caused by AS ultimately leads to the formation of new bones around the joints. Ossification in the joints and ligamentous structures of the vertebrae causes the formation of syndesmophytes, which can connect with each other allowing for specific radiographic findings [7-9]. 
In the spine, osteitis and sclerosis occur at the attachment of the annulus fibrosus to the vertebral bodies. Also, "squaring" due to erosion and remodeling of the vertebral body, provides definite findings. In later stages of the disease, ossification of the annulus fibrosus occurs, causing intervertebral bodies, bones, or syndesmophytes to form the so-called "bamboo spine." Apophysical joint falsification, ossification of spinal ligaments, and spondylodiscitis also occur [10].

MRI is primarily a more accurate detection measure in these cases, because radiographic findings may not be explicit until 2 years after the onset of the disease. The increased signal from bone and bone marrow in the MRI suggests osteitis and edema [11].

This paper reports the case of a 33-year-old female who, despite the sacroiliac joint being spared, had severe involvement of the spinal cord in the thoracic region, making the time order of bone involvement in this patient contrary to usual. The radiologic findings of AS for this patient will also be discussed and explained through literature review.

\section{Case Presentation}

A 33-year-old female referred to the rheumatology ward for chronic low back pain spanning 5 years. In the past, the patient had been treated with NSAID for the primary diagnosis of low back pain. In recent years, however, the

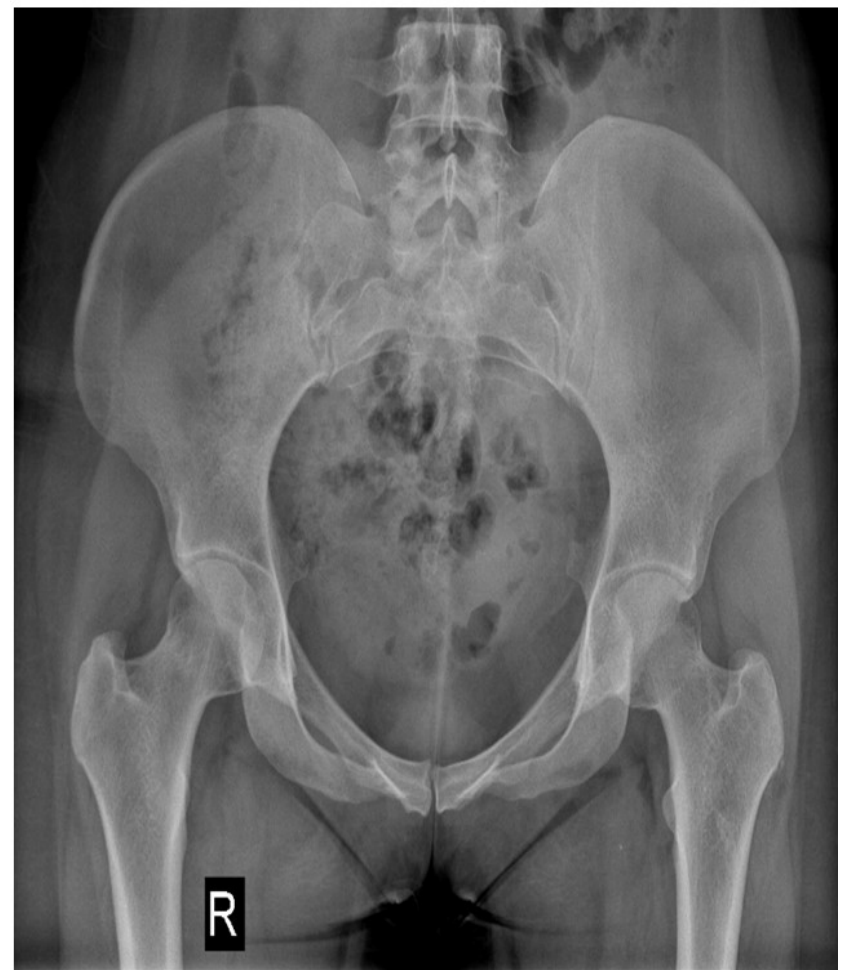

Figure 1. Abdominopelvic X-ray of patient; sacroiliac involvement is not seen. patient's physical activity gradually decreased, and she experienced morning stiffness lasting more than 30 minutes daily, which improved with movement and worsened with rest.

Throughout this period, the patient never experienced eye or GI problems (like uveitis or IBD), and renal function was always normal.

Physical examination revealed a negative Schober test (about $15 \mathrm{~cm}$ ), local tenderness over thoracic vertebrae 410 , but no restriction of movement. The patient had normal chest expansion, and no peripheral arthritis was seen.

Further investigations showed normal conditions: FBS: 98, Cr: 1, CBC: NL, ESR: $15 \mathrm{~mm} / \mathrm{h}$. CRP, Wright, and Coombs Wright, PPD were all negative as were HLA B27, anti-ds-DNA Ab, anticentromere $\mathrm{Ab}, \mathrm{Scl}-70 \mathrm{Ab}$, and anticardiolipin $\mathrm{Ab}$ (IgG- IgM). Electromyography of lower limbs and brain MRI were reported to be normal.

Unfortunately, the patient had experienced no improvement with NSAID treatment; thus, she scheduled appointments several times each year over the 5-year period. During these 5 years, lumbosacral X-rays were performed 3 times, and all of them were negative for the features of AS; the sacroiliac joint was also spared (Figure 1).

MRI findings for this patient revealed multiple inflammatory Schmorl's nodes, vertebral endplate inflammatory changes, and bone marrow edema (Figure 2).

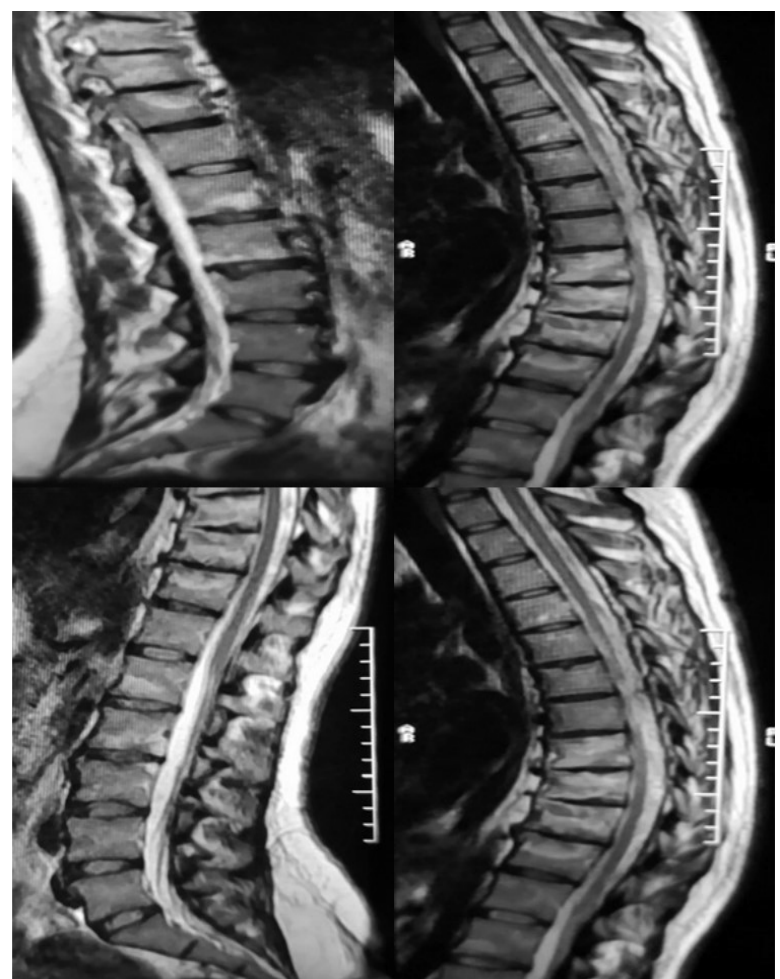

Figure 2. MRI of patient's spine; thoracic disc involvement demonstrates multiple inflammatory Schmorl's nodes, vertebral endplate inflammatory changes, and bone marrow edema. 
Treatment with NSAID was continued, but no significant clinical response was achieved. An abdominal $\mathrm{X}$-ray (Figure 3 ) and a control chest X-ray were performed, and amazing results were obtained; the patient had bamboo spine in the thoracic vertebrae (Figure 4). Based on the patient's inflammatory lower back pain, negative RF, and bamboo spine, the AS-appropriate anti-TNF therapy (etanercept $25 \mathrm{mg}$ twice weekly) was begun, and the patient's symptoms improved significantly.

This case presents many interesting educational points:
- According to the 1984 modified New York criteria for AS $[12,13]$, only 1-2 clinical criteria were meet, so this case did not match the AS definition; however, the patient had bamboo spine and responded appropriately to AS treatment.

- The sacroiliac joint was spared, even with severe thoracic involvement (i.e., bamboo spine).

- Joint involvement appeared in an unusual time order.

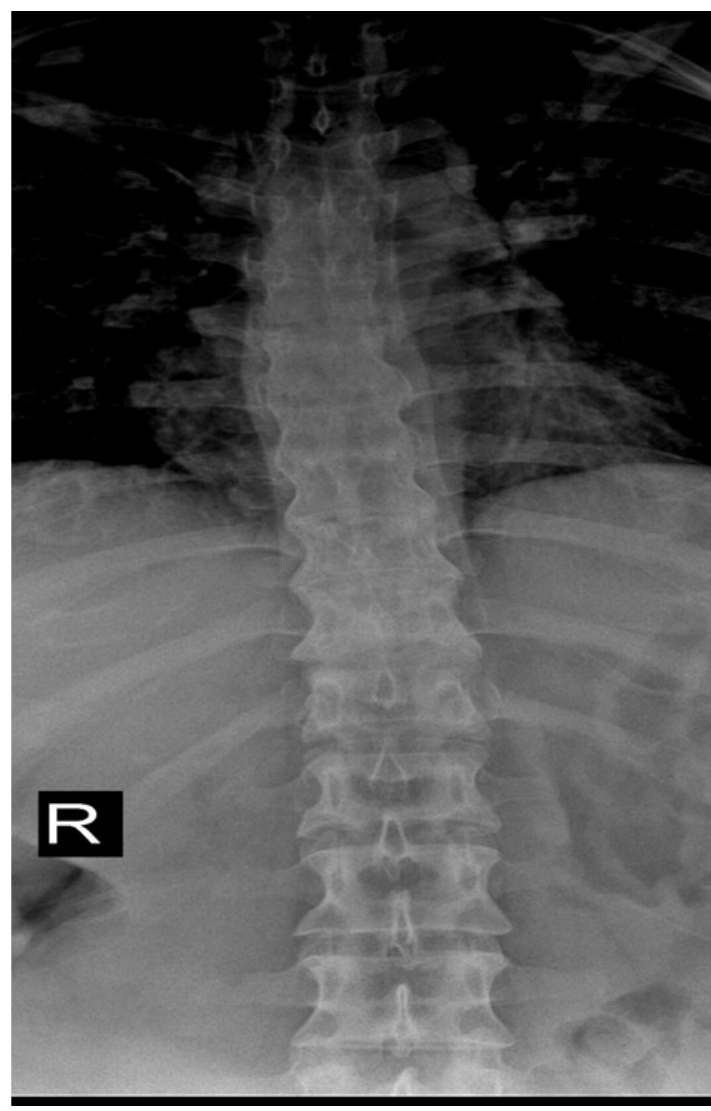

Figure 3. Abdominal X-ray of patient

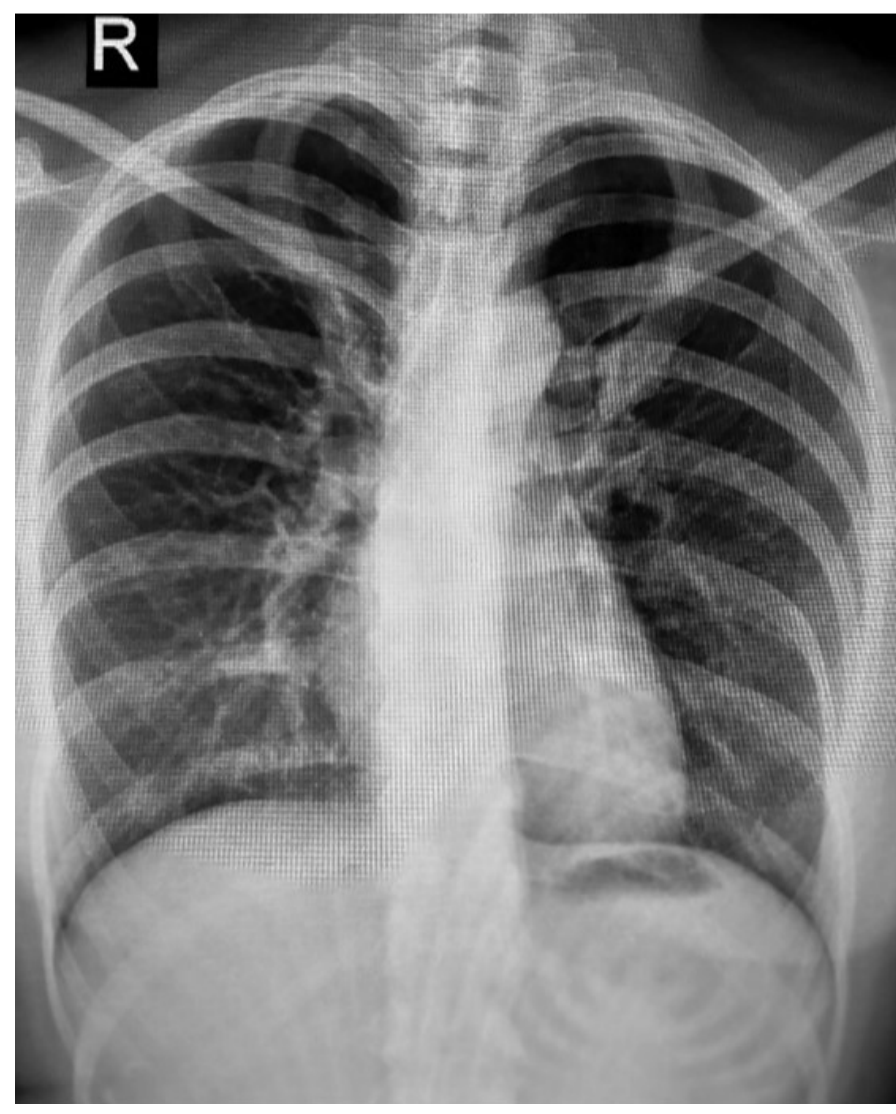

Figure 4. CXR of patient; bamboo spine in thoracic vertebrae is seen

\section{Discussion}

Spondyloarthropathy ( $\mathrm{SpA})$ includes a variety of diseases that sometimes overlap, including AS, psoriatic arthritis, reactive arthritis, arthritis/spondylitis associated with inflammatory bowel disease (IBD), and undifferentiated $\mathrm{SpA}$. The clinical manifestations of these diseases occur either predominantly in the axial or peripheral manner or as an overlap of these two patterns [14].

Ankylosing spondylitis is one of these diseases which affects the axial skeleton, although peripheral joints and even extra-articular structures can also be involved.

AS has a significant association with HLA-27; $90 \%$ of patients with AS are HLA-27 positive. Conversely, up to

$6 \%$ of patients who are genetically positive for HLA-27 are diagnosed with AS.

The hallmarks of ankylosing spondylitis (AS) are inflammation of the sacroiliac joints (SIJ) and spine usually accompanied by pain and stiffness of the body [15].

Histologically, the number of macrophages and osteoclasts increases in this disease, resulting in erosive joint edges, joint remodeling, and bone formation.

The first indicators of this disease in simple radiography are the disappearance of the cortical margins of the subchondral bone, subsequent wear, and bone sclerosis. Then, fibrosis and bone ankylosis also occur.

Radiographic sacroiliac joint evaluation is the most important diagnostic test for this disease. Although the diagnosis of spondyloarthritis can also be based on a 
patient's history and examination, a definitive diagnosis of AS requires radiographic findings.

In the past, the 1984 modified New York criteria for AS have been used to diagnose and classify AS. Radiographic changes in the sacroiliac joint were necessary to fulfill the diagnostic criteria for the disease, along with one of the clinical symptoms, such as morning stiffness that improved with activity but not with rest, or limited range of motion in the spine.

Since radiographic changes and limitations in spine motility are among the late findings of the disease, these criteria do not appear to be appropriate for diagnosis. Therefore, the necessary changes were made to the new criteria of AS diagnosis, where regardless of radiographic changes in the SI joint, the disease could be diagnosed [16].

In the current case, the sacroiliac joint was spared, so the radiological criteria were not met. As only 1-2 clinical criteria were meet, the case was not included in a definite diagnosis of AS. However, the patient also had a bamboo spine and responded appropriately to AS-appropriate treatment. This variety is one of the unique points of this report.

In many cases, it may take years for radiographic changes to occur in the sacroiliac joints of these patients; thus, MRI is increasingly being used to better diagnose these patients. Newer techniques are more sensitive in detecting early stages of intra-articular inflammation, cartilage changes, and bone marrow edema in sacroiliitis than simple radiography. They are also susceptible to acute and chronic changes in the spine [17].

Imaging has considerable specificity for AS (97.3\%), but its sensitivity is not high $(66.2 \%)$. Therefore, clinical features along with HLA-27 should be considered for the diagnosis of suspected patients, for example, chronic low back pain that begins at age 45 . The use of this genetic marker and clinical features increases the specificity of this criterion to $84.4 \%$ and its sensitivity to $82.9 \%$.

The anteroposterior pelvic view is sufficient for evaluating the inferior areas of the sacroiliac joint. The Ferguson view (AP with the tube angled 25 to 30 degrees cephalad) gives a complete view of the sacroiliac joint. Oblique views of any SI joint can also be obtained [18].

In the spinal column, inflammatory granulation tissue occurs in the vertebral bone and fibrous ring. The edges of the ring become eroded and eventually are replaced by bone; this is the beginning of syndesmophyte formation. As this process continues, the adjacent vertebral bodies connect to each other by forming a bone bridge: the socalled "bamboo spine" present in the spinal radiography.

Other findings that may be seen in vertebrates include disseminated osteoporosis, stiffness of the vertebral bodies on the margin of the disc, squares or barrels of the vertebrae, inflammation, and destruction of the border between the disc and the bone.

Early radiographic findings in this disease in lateral lumbar spine films resemble "shiny corners", or Romanus lesions, which are bone erosions in the corners of the vertebral bodies that are associated with bone resorption and reactive sclerosis. This process of inflammatory erosion and bone resorption results in vertebral bodies appearing "square" [19].

Romanus lesions are also seen in the chest and neck. Calcification and ossification of ligamentous structures between the trunks of the vertebrae cause the formation of syndesmophytes, which are present in the X-ray.

Generally, in the thoracolumbar spine, as a result of the fusion of syndesmophytes around the annulus fibrosis, a long column that resembles a bamboo stem is formed. Romanus lesions are an early finding in the spine of AS patients resulting from the erosion of the annulus fibrosus junction at the anterior edge of the vertebral body endplates $[20,21]$. As the inflammatory process progresses, sclerosis causes a "shiny corner". As the spinous processes become bony, a "dagger sign" is formed [22].

The apophyseal joints of the spine may become fused, resulting in limited motility.

Osteophytes, which are commonly found in the right thoracic spine but also seen in the neck and lumbar regions, are also a symptom of diffused idiopathic skeletal hyperostosis (DISH), a disease with similar features to AS. DISH is usually differentiated with AS by features such as lack of sacroiliitis as well as a late-onset detection of the disease [23].

Importantly, there are some differences between usual syndesmophyte and bamboo spine. For example:

- Usual syndesmophyte is the ossification of osteophytes. This syndesmophyte has a sharper angle and is spindle-shaped, unlike bamboo spine.

- Bamboo spine is ossification of the annulus fibrosus + anterior longitudinal ligaments in the intervertebral discs. A marginal syndesmophyte between adjoining vertebral bodies will be created. The resulting radiographic appearance is thin, curved, radiopaque spicules that completely bridge adjoining vertebral bodies. As we see in the current patient's X-ray, the curved and bridging bamboo spine is obvious in the thoracic vertebrae, especially above the diaphragm (Figure 1).

Syndesmophytes are commonly found in the thoracic region, regardless of whether they are present in the lumbar region. Moreover, bridging between the trunks of the vertebrae occurs most commonly in the mid-thoracic region, which may be due to limited movement or height of the disc in this region.

It is important to note that typically, if syndesmophytes are present in the lumbar spine, one can expect to see them in the thoracic region; however, this is not always the case. Several patients have been found to have a normal lumbar $\mathrm{X}$-ray, but in their thoracic regions, syndesmophytes have been seen. Therefore, syndesmophytes are more common in the thoracic regions, and T11-L2 is the most frequently reported area affected [24, 25].

In $\mathrm{AS}$, the distribution of syndesmophytes also varies in the thoracic and lumbar spine. In a study of 18 patients with AS, syndesmophytes were seen in the thoracic discs of 65 $81 \%$, lumbar spine in $56-72 \%$, and at the thoracolumbar 
junction in $83-89 \%$ of participants [26]. Bridging was more common in the thoracic discs, probably due to narrower disc spaces or less motion. Usually, cases with lumbar syndesmophytes also had thoracic syndesmophytes, but in 9 cases with thoracic syndesmophytes, there were few or there was no lumbar involvement. This pattern suggests that thoracic syndesmophytes typically develop before lumbar syndes-mophytes.

Diagnosing AS from abdominal X-rays is also important, because in the late stages of the disease the spinal column becomes very fragile, and even the slightest trauma can cause a significant fracture.

\section{Conclusion}

Clinical and laboratory data showed considerable disease control, however, relapse of disease was seen dueOverall, the involvement of the thoracic spine in ankylosing spondylitis can result in "bamboo spine," which is a rare manifestation. "Bamboo spine" is one of the late symptoms of the disease; however, the patient in this case study had not been diagnosed for 5 years, even with thoracic involvement; it took five years to stabilize the radiological changes. The uniqueness of this patient lies in the fact that her thoracic is involved (in a bamboo pattern), but her lumbar and sacroiliac joint was spared. This finding is contrary to the usual time course of AS disease and is of educational importance for early diagnosis of AS. The radiographic changes, coupled with other clinical manifestations of the patient, are an interesting element of the current case.

Radiologic manifestation in rheumatologic disease may occur without any predictable order; for example, the first radiologic presentation of the current patient was the bamboo spine. Paying attention to the course of the disease as well as the clinical findings and various imaging results of the different areas (heart, lumbar spine, and sacroiliac joint) will help physicians diagnose the disease as soon as possible. It can also reduce the burden of complications and assist physicians in beginning treatment at the right time.

\section{Conflict of interest}

The authors report that they have no conflicts of interest to declare.

\section{References}

1. Miller MM. Ankylosing spondylitis, Reiter's syndrome, psoriatic arthritis, and arthritis of inflammatory bowel disease. Prim Care 1984; 11(2):271-82.

2. Tam LS, Wei JC, Aggarwal A, Baek HJ, Cheung PP, Chiowchanwisawakit P. et al. 2018 APLAR axial spondyloarthritis treatment recommendations. Int $\boldsymbol{J}$ Rheum Dis 2019; 22(3):340-56. doi: 10.1111/1756185X.13510.

3. Smolen JS, Schols M, Braun J, Dougados M, FitzGerald O, Gladman DD. et al. Treating axial spondyloarthritis and peripheral spondyloarthritis, especially psoriatic arthritis, to target: 2017 update of recommendations by an international task force. Ann Rheum Dis 2018; 77(1):3-17. doi: 10.1136/annrheumdis-2017-211734.

4. van Denderen JC, van der Paardt M, Nurmohamed MT, de Ryck YMMA, Dijkmans BAC, van der HorstBruinsma IE. Double blind, randomised, placebo controlled study of leflunomide in the treatment of active ankylosing spondylitis. Ann Rheum Dis 2005; 64(12):1761-64. doi: 10.1136/ard.2005.036491.

5. Sieper J, Braun J, Rudwaleit M, Boonen A, Zink A. Ankylosing spondylitis: an overview. Ann Rheum Dis 2002; 61 Suppl 3(Suppl 3):iii8-18. doi: 10.1136/ard.61.suppl_3.iii8.

6. Zink A, Braun J, Listing J, Wollenhaupt J. Disability and handicap in rheumatoid arthritis and ankylosing spondylitis--results from the German rheumatological database. German Collaborative Arthritis Centers. J Rheumatol 2000; 27(3):613-22.
7. Weisman MH. Inflammatory back pain: the United States perspective. Rheum Dis Clin North Am 2012; 38(3):501-12. doi: 10.1016/j.rdc.2012.09.002.

8. Braun J, Inman R. Clinical significance of inflammatory back pain for diagnosis and screening of patients with axial spondyloarthritis. Ann Rheum Dis 2010; 69(7):1264-68. doi: 10.1136/ard.2010.130559.

9. Thomas GP, Duan R, Pettit AR, Weedon H, Kaur S, Smith M. et al. Expression profiling in spondyloarthropathy synovial biopsies highlights changes in expression of inflammatory genes in conjunction with tissue remodelling genes. BMC Musculoskelet Disord 2013; 14:354. doi: 10.1186/14712474-14-354.

10. Braun J, van den Berg R, Baraliakos X, Boehm H, Burgos-Vargas R, Collantes-Estevez E. et al. 2010 update of the ASAS/EULAR recommendations for the management of ankylosing spondylitis. Ann Rheum Dis 2011; 70(6):896-04. doi: 10.1136/ard.2011.151027.

11. Guellec D, Nocturne G, Tatar Z, Pham T, Sellam J, Cantagrel A. et al. Should non-steroidal antiinflammatory drugs be used continuously in ankylosing spondylitis? Joint Bone Spine 2014; 81(4):308-12. doi: 10.1016/j.jbspin.2014.01.003.

12. Akgul O, Ozgocmen S. Classification criteria for spondyloarthropathies. World J Orthop 2011; 2(12):107-15. doi: 10.5312/wjo.v2.i12.07. 
13. van der Linden S, Valkenburg HA, Cats A. Evaluation of diagnostic criteria for ankylosing spondylitis. A proposal for modification of the New York criteria. Arthritis Rheum 1984; 27(4):361-68. doi: 10.1002/art.1780270401.

14. Dougados M, Baeten D. Spondyloarthritis. Lancet 2011; 377(9783):2127-37. doi: 10.1016/S01406736(11)60071-8.

15. Braun J, Sieper J. Ankylosing spondylitis. Lancet 2007; 369(9570):1379-90. doi: $10.1016 / \mathrm{S} 0140-$ 6736(07)60635-7.

16. Rudwaleit M, van der Heijde D, Landewe R, Listing J, Akkoc N, Brandt J. et al. The development of Assessment of SpondyloArthritis international Society classification criteria for axial spondyloarthritis (part II): validation and final selection. Ann Rheum Dis 2009; 68(6):777-83. doi: 10.1136/ard.2009.108233.

17. Hermann K-GA, Althoff CE, Schneider U, Zühlsdorf S, Lembcke A, Hamm B. et al. Spinal Changes in Patients with Spondyloarthritis: Comparison of MR Imaging and Radiographic Appearances. Radiographics 2005; 25(3):559-69. doi: 10.1148/rg.253045117.

18. Garrett S, Jenkinson T, Kennedy LG, Whitelock H, Gaisford P, Calin A. A new approach to defining disease status in ankylosing spondylitis: the Bath Ankylosing Spondylitis Disease Activity Index. J Rheumatol 1994; 21(12):2286-91.

19. Sandborn WJ, Stenson WF, Brynskov J, Lorenz RG, Steidle GM, Robbins JL. et al. Safety of celecoxib in patients with ulcerative colitis in remission: a randomized, placebo-controlled, pilot study. Clin Gastroenterol Hepatol 2006; 4(2):203-11. doi: 10.1016/j.cgh.2005.12.002.

20. Ostergaard M, Lambert RGW. Imaging in ankylosing spondylitis. Ther Adv Musculoskelet Dis 2012; 4(4):301-11. doi: 10.1177/1759720X11436240.
21. Hermann KG, Althoff CE, Schneider U, Zuhlsdorf S, Lembcke A, Hamm B. et al. Spinal changes in patients with spondyloarthritis: comparison of MR imaging and radiographic appearances. Radiographics 2005; 25(3):559-69. doi: 10.1148/rg.253045117.

22. Yasin Y, Perumpillichira J, Dyer RB. Classics in abdominal imaging: the bamboo spine. Abdom Radiol (NY) 2016; 41(10):2078-79. doi: 10.1007/s00261-0160784-x.

23. Reveille JD, Arnett FC. Spondyloarthritis: update on pathogenesis and management. Am J Med 2005; 118(6):592-03. doi: 10.1016/j.amjmed.2005.01.001.

24. Braun J, Baraliakos X, Golder W, Hermann K-G, Listing $\mathrm{J}$, Brandt $\mathrm{J}$. et al. Analysing chronic spinal changes in ankylosing spondylitis: a systematic comparison of conventional $\mathrm{x}$ rays with magnetic resonance imaging using established and new scoring systems. Ann Rheum Dis 2004; 63(9):1046-55. doi: 10.1136/ard.2003.019968.

25. Baraliakos X, Listing J, Rudwaleit M, Sieper J, Braun J. Development of a radiographic scoring tool for ankylosing spondylitis only based on bone formation: addition of the thoracic spine improves sensitivity to change. Arthritis Rheum 2009; 61(6):764-71. doi: 10.1002/art.24425.

26. Tan S, Yao L, Ward MM. Thoracic Syndesmophytes Commonly Occur in the Absence of Lumbar Syndesmophytes in Ankylosing Spondylitis: A Computed Tomography Study. J Rheumatol 2017; 44(12):1828-32. doi: 10.3899/jrheum. 170340. 